By way of a

\title{
FOREWORD
}

In 1933, John von Neumann [28 December 1903-8 February 1957] became (together with Albert Einstein and Oswald Veblin) a member of the founding faculty (and Kurt Gödel a visiting member) of the Institute for Advanced Study, where he remained for the remainder of his life. The distinguished author of the following commemorative essay was a member in 1949 and 1952-1954, and had then an opportunity to become personally acquainted with von Neumann. The essay was taken (with permission) from volume 64, number 3, part 2 (May 1958) pages 95-99 of the Bulletin of the American Mathematical Society, the 129-page entirety of which is given over to expert surveys of von Neumann's contributions to eight different subject areas.

\section{VON NEUMANN'S CONTRIBUTIONS TO QUANTUM THEORY}

\author{
LÉON VAN HOVE
}

That von Neumann has been "par excellence" the mathematician of quantum mechanics is as obvious to every physicist now as it was a quarter of a century ago. Quantum mechanics was very fortunate indeed to attract, in the very first years after its discovery in 1925, the interest of a mathematical genius of von Neumann's stature. As a result, the mathematical framework of the theory was developed and the formal aspects of its entirely novel rules of interpretation were analyzed by one single man in two years time (1927-1929). Conversely, one could almost say in reciprocity, quantum mechanics introduced von Neumann into a field of mathematical investigation, operator theory, in which he achieved some of his most prominent successes.

Von Neumann's major contributions to quantum mechanics are his development of the mathematical framework of the theory and his formal study of quantum statistics, quantum measuring processes and their interrelations. Whereas the latter study was essentially complete in 1927 (except for the quantum ergodic theorem of 1929) the work on the mathematical foundations of quantum mechanics came to its culmination in 1929 with the spectral theorem 
for hypermaximal symmetric operators in Hilbert space. In the next two paragraphs we shall discuss these major contributions.

The mathematical framework of quantum theory. By the time von Neumann started his investigations on the formal framework of quantum mechanics this theory was known in two different mathematical formulations: the "matrix mechanics" of Heisenberg, Born and Jordan, and the "wave mechanics" of Schrödinger. The mathematical equivalence of these formulations had been established by Schrödinger, and they had both been embedded as special cases in a general formalism, often called the "transformation theory," developed by Dirac and Jordan. This formalism, however, was rather clumsy and it was hampered by its reliance upon ill-defined mathematical objects, the famous delta-functions of Dirac and their derivatives. Although von Neumann himself attempted at first, in collaboration with Hilbert and Nordheim [1], to edify the formalism along similar lines, he soon realized that a much more natural framework was provided by the abstract, axiomatic theory of Hilbert spaces and their linear operators [2]. This mathematical formulation of quantum mechanics, whereby states of the physical system are described by Hilbert space vectors and measurable quantities by Hermitian operators acting upon them, has been very successful indeed. Unchanged in its essentials it has survived the two great extensions which quantum theory was to undergo soon: the relativistic quantum mechanics of particles (Dirac equation) and the quantum theory of fields.

One might of course remark that Dirac's delta functions and their derivatives, although poorly defined at the time of their introduction, have been recognized since as bona fide mathematical entities in L. Schwartz' theory of distributions. This is quite true and moreover these functions have been used continually by physicists throughout the development of quantum theory, in particular in the last two decades for the study of scattering processes and of quantized fields. Delta functions have established themselves as the natural tool whenever operators with continuous spectra are to be considered. This does not affect in any way, however, the fact that the axiomatically defined separable Hilbert space is the suitable framework for the quantum-mechanical formalism as we know it today, and the recognition of this fact we owe to von Neumann.

An essential feature of the Hilbert space formulation of quantum theory is that the most important physical quantities (such as position, momentum or energy) are represented by unbounded Hermitian operators. Since the theoretical prediction of measurements makes essential use of the spectral resolution of the operators representing the physical quantities, von Neumann was, in his very first invetigation [2], faced with the problem of extending to the unbounded case the known spectral theory of bounded Hermitian operators. By 1929 he had brought this problem to a complete solution [3]. He introduced the all-important concept of hypermaximal symmetric operator, being the most general Hermitian operator with a spectral resolution. This work, the results of which were reached independently by M. H. Stone [4], was for von Neumann 
the start of a long series of investigations on linear operators in Hilbert space.

Still another contribution of von Neumann to the mathematical foundation of quantum theory is worth mentioning here. He established the important theorem that (in the irreducible case and after a suitable reformulation) the canonical commutation rules $Q_{j} P_{\ell}-P_{\ell} Q_{j}=\hbar i \delta_{j \ell}$ determine the operators $Q_{1}, \ldots, Q_{n}, P_{1}, \ldots, P_{n}$ uniquely except for an arbitrary transformation [5]. Although rarely quoted as such, this theorem, which was already known to Dirac and Stone [6], is really fundamental for the understanding of many quantummechanical investigations where the theoretical analysis is exclusively based on the canonical commutation rules or in the equivalent field-theoretical form

$$
A_{j} A_{\ell}^{*}-A_{\ell}^{*} A_{j}=\hbar \delta_{j \ell}, \quad 2^{\frac{1}{2}} A_{j}=P_{j}-i Q_{j}
$$

Statistical aspects of quantum theory. In the course of his formulation of quantum mechanics in terms of vectors and operators of Hilbert space von Neumann also gave in complete generality the basic statistical rule of interpretation of the theory. This rule concerns the result of the measurement of a given physical quantity on a system in a given quantum state and expresses its probability distribution by means of a simple and now completely familiar formula involving the vector representing the state and the spectral resolution of the operator which represents the physical quantity [2]. This rule, originally proposed by Born in 1926, was for von Neumann the starting point of a mathematical analysis of quantum mechanics in entirely probabilistic terms. The analysis, carried out in a paper of 1927 [7], introduced the concept of statistical matrix for the description of an ensemble of systems which are not necessarily all in the same quantum state. The statistical matrix (now often called $\rho$-matrix although von Neumann's notation was $U$ ) has become one of the major tools of quantum statistics and it is through this contribution that von Neumann's name became familiar to even the least mathematically minded physicists.

In the same paper von Neumann also investigates a problem which is still now the subject of much discussion; viz., the theoretical description of the quantum-mechanical measuring process and of the non-causal elements which it involves. Mathematically speaking von Neumann's study of this delicate question is quite elegant. It provides a clear-cut formal framework for the numerous investigations which were needed to clarify physically the all-important implications of quantum phenomena for the nature of physical measurements, the most important of which is Neils Bohr's concept of complementarity.

The results of the paper just discussed were immediately used by the author to lay the foundation for quantum thermodynamics [8]. He gave the quantum analogue

$$
S=-k \operatorname{tr}(\rho \ln \rho), \quad \rho \text { denotes a statistical matrix }
$$

of the well known classical formula for the entropy

$$
S=-k \int f \ln f d \omega, \quad f \text { denotes a distribution function in phase space. }
$$


He further wrote down the density matrix for a canonical ensemble at temperature $T$ :

$$
\rho=Z^{-1} \exp (-H / k T), \quad Z=\operatorname{tr}[\exp (-H / k T)],
$$

$H$ being the Hamiltonian operator. Two years later von Neumann came back to quantum thermodynamics with a contribution to a much more difficult problem: the formulation and proof of an ergodic theorem for quantum systems [9]. The basic principle of this work is to define quantum analogues of cells in phase space by considering sets of quantum states for which all macroscopic quantities have given values with a certain inaccuracy. One further considers the unitary transformation $u$ relating these quantum states to the eigenstates of the Hamiltonian. The ergodicity is then established for "almost every" value of the transformation $u$. Although the latter restriction is a rather unsatisfactory one from a physical standpont, one must consider von Neumann's ergodic theorem as one of the very few important contributions to a most difficult subject which even now is far from complete clarification.

Most of the work we have briefly reviewed has been republished by the author, in greatly expanded form, as a book which rapidly became and still is the standard work on the mathematical foundations of quantum mechanics [10], [and of which the present volume is an English translation]. Von Neumann devoted in his book considerable attention to a point which had not been discussed in the 1927 papers and which was later the subject of much controversy. It is the question of the possible existence of "hidden variables," the consideration of which would eliminate the non-causal element involved in the measurement process. Von Neumann could show that hidden parameters with this property cannot exist if the basic structure of quantum theory is retained. Although he mentioned the latter restriction explicitly, $\dagger$ his result is often quoted without due reference to it, a fact which sometimes gave rise to unjustified criticism in the many discussions devoted through the years to the possibility of an entirely deterministic reformulation of quantum theory.

Other contributions. As von Neumann's complete bibilography will reveal, he wrote quite a few other papers on quantum mechanics, often in collaboration with physicists, especially with Wigner. Most of these papers deal with technical matters and the importance of the major contributions discussed above is so eminent that, in comparison, the other papers' scope is modest. There is only one broad subject which we would like to mention here, because von Neumann, obviously giving it considerable thought, returned to it several times in 1934 and 1936 (in collaboration with Jordan, Wigner and Garrett Birkhoff). It is the question of the algebraic and logical structure of quantum mechanics, where the hope has existed to reach through abstract analysis possible generalizations of the accepted theory. Nobody knows whether such a hope is justified, but it is undoubtedly a natural one and it has appealed to many other people, giving one more example of the power and originality of von Neumann's thinking.

$\dagger$ See, e.g., [10], p. 109, line 17 et seq [or below: p. 132, paragraph 3]. 
1. D. Hilbert, J. von Neumann \& L. Nordheim, Über die Grundlagen der Quantenmechanik, Math. Ann. vol. 98 (1927) pp. 1-30.

2. J. von Neumann, Mathematische Begründung der Quantenmechanik, Nachr. Ges. Wiss. Göttingen (1927), pp. 1-57.

3.—, Allgemeine Eigenwerttheorie Hermitischer Functionaloperatoren, Math. Ann. vol. 102 (1929) pp. 49-131.

4. M. H. Stone, Linear transformations in Hilbert space and their applications to analysis, Amer. Math. Soc. Colloquium Publications, vol. 15, 1932.

5. J. von Neumann, Die Eindeutigkeit der Schrödingerschen Operatoren, Math. Ann. vol. 104 (1931) pp. 570-578.

6. P. A. M. Dirac, The Principles of Quantum Mechanics, Oxford, Clarendon Press (1930), $\S 29$.

M. H. Stone, Linear transformations in Hilbert space. Operational methods and group theory, Proc. Nat. Acad. Sci. U.S.A. vol. 16 (1930) pp. 172-175.

7. J. von Neumann, Wahrscheinlichkeitstheoretischer Aufbau der Quantenmechanik, Nachr. Ges. Wiss. Göttingen (1927) pp. 245-272.

8. — , Thermodynamik quantenmechanischer Gesamtheiten, Nachr. Ges. Wiss. Göttingen (1927) pp. 273-291.

9. - Beweis des Ergodensatzes und des H-Theorems in der neuen Mechanik, Zeitschrift für Physik, vol. 57 (1929) pp. 30-70.

10. - Mathematische Grundlagen der Quantenmechanik, Berlin, J. Springer (1932).

Institute for Theoretical Physics of the University, Utrecht, NetherLANDS

A "von Neumann Days" Symposium took place on 3-7 May 2010 at Brown University's Center for Computational Molcular Biology. Remarks prepared for presentation on that occasion by Freeman Dyson-who was a member of the Institute for Advanced Study in 1948-1949 and became there a tenured faculty colleague of von Neumann in 1953-were subsequently published as "A walk through Johnny von Neumann's garden" (Notices of the AMS 60, 155-161 (2013)), from which the following excerpt has (with permission) been taken.

\section{VON NEUMANN WORK RELATED TO QUANTUM THEORY}

\section{FREEMAN DYSON}

During his Berlin years, Johnny made frequent visits to Göttingen, where Heisenberg had recently invented quantum mechanics and Hilbert was the presiding mathematician. Hilbert was intensely interested in quantum mechanics and encouraged collaboration between mathematicians and physicists. From the point of view of Hilbert, quantum mechanics was a mess. Heisenberg had no use for rigorous mathematics and no wish to learn it. Dirac made free use of his 
famous delta-function, which was defined by a mathematical absurdity, being infinite at a single point and zero everywhere else. When Hilbert remarked to Dirac that the delta-function could lead to mathematical contradictions, Dirac replied, "Did I get into mathematical contradictions?" Dirac knew that his delta-function was a good tool for calculating quantum processes, and that was all that he needed. Twenty years later, Laurent Schwartz provided a rigorous basis for the delta-function and proved that Dirac was right. Meanwhile, Johnny worked with Hilbert and published a series of papers cleaning up the mess. In 1932 he published the book Mathematical Foundations of Quantum Mechanics [9], which occupies a substantial piece of his garden.

Johnny's book was the first exposition of quantum mechanics that made the theory mathematically respectable. The concepts were rigorously defined and the consequences rigorously deduced. Much of the work was original, especially the chapters on quantum statistics and the theory of measurement. I read the book in 1946 when I was still a pure mathematician but already intending to switch my attention to physics. I found it enormously helpful. It gave me what I needed, a mathematically precise statement of the theory, explaining the fine points that the physicists had been too sloppy to mention. From that book I learned most of what I know about quantum mechanics. But then, after I had made the transition to physics and had begun to read the current physics journals, I was surprised to discover that nobody in the physics journals ever referred to Johnny's book. So far as the physicists were concerned, Johnny did not exist. Of course, their ignorance of Johnny's work was partly a problem of language. The book was in German, and the first English translation was only published in 1955. But I think even if the book had been available in English, the physicists of the 1940s would not have found it interesting. That was a time when the culture of physics and the culture of mathematics were most widely separated. The culture of physics was dominated by people like Oppenheimer who made friends with poets and art historians but not with pure mathematicians. The culture of mathematics was dominated by the Borbaki cabal, which tried to expunge from mathematics everything that was not purely abstract. The gap between physics and mathematics was as wide as the gap between science and the humanities described by C. P. Snow in his famous lecture on the two cultures. Johnny was one of the very few people who were at home in all four cultures: in physics and mathematics, but also in science and the humanities.

The central concept in Johnny's version of quantum mechanics is the abstract Hilbert space. Hilbert space is the infinite-dimensional space in which quantum states are vectors and observable quantities are linear operators. Hilbert had defined and explored Hilbert space long before quantum mechanics made it useful. The unexpected usefulness of Hilbert space arises from the fact that the equations of quantum mechanics are exactly linear. The operators form a linear algebra, and the states can be arranged in multiplets defined by linear representations of the algebra. Johnny liked to formulate physical problems in abstract and general language, so he formulated quantum mechanics as a theory 
of rings of linear operators in Hilbert space. A ring means a set of operators that can be added or subtracted or multiplied together but not divided. Any physical system obeying the rules of quantum mechanics can be described by a ring of operators. Johnny began studying rings of operators to find out how many different types of quantum systems could exist.

After Johnny published his quantum mechanics book, he continued for several years to develop the theory of rings of operators. The third volume of his collected works consists entirely of papers on rings of operators. $\mathrm{He}$ published seven long papers with a total of more than five hundred pages. I will not discuss these monumental papers this morning. They contain Johnny's deepest work as a pure mathematician. He proved that every ring of operators is a direct product of irreducible rings that he called factors. He discovered that there are five types of factor, of which only two were previously known. Each of the types has unique and unexpected properties. Exploring the ocean of rings of operators, he found new continents that he had no time to survey in detail. He left the study of the three new types of factor unfinished. He intended one day to publish a grand synthesis of his work on rings of operators. The grand synthesis remains an unwritten masterpiece, like the eighth symphony of Sibelius.

The quantum mechanics book is the last item on my list of flowers that Johnny published in German. It was published in 1932 when he was dividing his time equally between Berlin and Princeton. In the same year he began writing papers in English. One of his first papers to appear in English was "Proof of the quasi-ergodic hypothesis" [10], which he published in the Proceedings of the National Academy of Sciences to make sure that American mathematicians would read it. This paper solved an important problem in classical mechanics using the same concept of Hilbert space that he had used to solve problems in quantum mechanics. A classical dynamical system is said to be ergodic if after we put it into an initial state and then leave it alone for an infinite time, it comes arbitrarily close to any final state with probability independent of the initial state. Johnny proved that under certain clearly specified conditions, a system is ergodic if and only if there exist no constants of the motion. A constant of the motion means a quantity depending on the state of the system which does not change as the system moves forward in time. Johnny's theorem provides a firm mathematical basis for the assumptions that are customarily made by physicists using classical statistical mechanics. Translated into the sloppy language used by physicists, the theorem says that the time-average of any single trajectory of the system over a long time is equal to the statistical average of all trajectories. Even more sloppily, physicists say that time-averages are equal to ensemble averages, and we use the word ensemble to mean the set of all states of the system.

One of the American mathematicians who read Johnny's paper in the Proceedings of the National Academy of Sciences was Garrett Birkhoff. Garrett was the son of George Birkhoff, and both father and son were famous mathematicians. Garrett and Johnny became close friends, and Garrett came 
to Princeton for frequent visits. After Johnny died, Garrett wrote a memoir about the work that Johnny did in the 1930s. Here is a sentence from Garrett's memoir: "Anyone wishing to get an unforgettable impression of the razor edge of von Neumann's mind need merely try to pursue this chain of exact reasoning for himself, realizing that often five pages of it were written down before breakfast, seated at a living room writing-table in a bathrobe."

REFERENCES

9. J. von Neumann, Mathematische Grundlagen der Quantenmechanik, Springer, Berlin, 1932.

10. - "Proof of the quasi-ergodic hypothesis," Proc. Nat. Acad. Sci. 18, 70-82 (1932).

Institute for Advanced Studies, Princeton 
MATHEMATICAL FOUNDATIONS OF QUANTUM MECHANICS 
